Kruszka Paul (Orcid ID: 0000-0003-4949-0875)

Bedeschi Maria Francesca (Orcid ID: 0000-0003-0174-3562)

Martinez-Agosto Julian (Orcid ID: 0000-0001-6776-6949)

Boycott Kym (Orcid ID: 0000-0003-4186-8052)

\title{
Phenotype Delineation of ZNF462 related syndrome
}

Paul Kruszka ${ }^{1}$, Tommy Hu${ }^{1}$, Sungkook Hong ${ }^{1}$, Rebecca Signer ${ }^{2}$, Benjamin Cogné ${ }^{3}$, Betrand Isidor $^{3}$, Sarah E. Mazzola ${ }^{4}$, Jacques C. Giltay ${ }^{5}$, Koen L.I. van Gassen ${ }^{5}$, Eleina M. England ${ }^{6}$, Charlotte W. Ockeloen ${ }^{7}$, Pedro A. Sanchez-Lara ${ }^{8,9}$, Esther Kinning ${ }^{10}$, Darius J Adams ${ }^{11}$, Kayla Treat ${ }^{12}$, Wilfredo Torres-Martinez ${ }^{12}$, Maria Francesca Bedeschi ${ }^{13}$, Maria lascone ${ }^{14}$, Stephanie Blaney ${ }^{15}$, Oliver Bell17, Tiong Y. $\operatorname{Tan}^{18,19,20}$, Marie-Ange Delrue ${ }^{21}$, Julie Jurgens ${ }^{22}$, Brenda J. Barry ${ }^{6,23}$, Elizabeth C. Engle $^{6,23,24}$, Sarah K. Savage ${ }^{25}$, Nicole Fleischer ${ }^{25}$, Julian A. Martinez-Agosto ${ }^{2}$, Kym Boycott $^{16}$, Elaine H. Zackai ${ }^{4}$, Maximilian Muenke ${ }^{1}$

1. Medical Genetics Branch, National Human Genome Research Institute, National Institutes of Health, Bethesda, MD, USA

2. Department of Human Genetics, David Geffen School of Medicine, University of California, Los Angeles, CA, USA

3. Service de génétique médicale, Hôtel-Dieu, CHU de Nantes, Nantes, France

4. Division of Human Genetics, The Children's Hospital of Philadelphia, Philadelphia, PA, USA

5. Department of Genetics, University Medical Center Utrecht, Utrecht, The Netherlands

6. Center for Mendelian Genomics, Broad Institute of MIT and Harvard, Cambridge, Massachusetts, USA

7. Department of Human Genetics, Radboud University Medical Center, Nijmegen, the Netherlands.

8. Keck School of Medicine, University of Southern California, Los Angeles, CA, USA

9. Department of Pediatrics, David Geffen School of Medicine, University of California, Los Angeles, CA, USA.

This is the author manuscript accepted for publication and has undergone full peer review but has not been through the copyediting, typesetting, pagination and proofreading process, which may lead to differences between this version and the Version of Record. Please cite this article as doi: 10.1002/ajmg.a.61306

This article is protected by copyright. All rights reserved. 
10. West of Scotland Genetics Service, Queen Elizabeth Hospitals, Glasgow, Scotland

11. Atlantic Health System, Morristown, NJ, USA

12. Indiana University School of Medicine, Indianapolis, IN, USA

13. Medical Genetic Unit, Fondazione IRCCS Ca' Granda Ospedale Maggiore Policlinico, Milan, Italy

14. Laboratorio di Genetica Medica, ASST Papa Giovanni XXIII, Bergamo, Italy

15. Algoma Public Health, Sault Ste Marie, ON, Canada

16. Children's Hospital of Eastern Ontario Research Institute, University of Ottawa, Ottawa, ON, Canada.

17. Keck School of Medicine, University of Southern California, Los Angeles, CA

18. Murdoch Children's Research Institute, Melbourne, Australia.

19. Department of Paediatrics, University of Melbourne, Melbourne, Australia

20. Victorian Clinical Genetics Services, Melbourne, Australia

21. Département de pédiatrie, Service de génétique médicale, Centre Hospitalier Universitaire Ste-Justine, Université de Montréal, Montréal, Canada

22. Department of Neurology, Boston Children's Hospital, Harvard Medical School, Boston, MA, USA

23. Howard Hughes Medical Institute, Chevy Chase, MD

24. Department of Ophthalmology, Boston Children's Hospital, Harvard Medical School, Boston, MA

25. FDNA Inc., Boston, MA, USA

This article is protected by copyright. All rights reserved. 
Correspondence: Paul Kruszka. paul.kruszka@nih.gov

This article is protected by copyright. All rights reserved. 


\section{ABSTRACT}

Zinc finger protein 462 (ZNF462) is a relatively newly discovered vertebrate specific protein with known critical roles in embryonic development in animal models. Two case reports and a case series study have described the phenotype of 10 individuals with ZNF462 loss of function variants. Herein, we present 14 new individuals with loss of function variants to the previous studies to delineate the syndrome of loss of function in ZNF462. Collectively, these 24 individuals present with recurring phenotypes that define a multiple congenital anomaly syndrome. Most have some form of developmental delay (79\%) and a minority have autism spectrum disorder (33\%). Characteristic facial features include ptosis (83\%), down slanting palpebral fissures (58\%), exaggerated Cupid's bow/wide philtrum (54\%), and arched eyebrows (50\%). Metopic ridging or craniosynostosis was found in a third of study participants

and feeding problems in half. Other phenotype characteristics include dysgenesis of the corpus callosum in $25 \%$ of individuals, hypotonia in half, and structural heart defects in $21 \%$. Using facial analysis technology, a computer algorithm applying deep learning was able to accurately differentiate individuals with ZNF462 loss of function variants from individuals with Noonan syndrome and healthy controls. In summary, we describe a multiple congenital anomaly syndrome associated with haploinsufficiency of ZNF462 that has distinct clinical characteristics and facial features. 


\section{KEYWORDS}

ZNF462, ptosis, developmental delay, autism spectrum disorders, corpus callosum, craniosynostosis

This article is protected by copyright. All rights reserved. 


\section{INTRODUCTION}

Heterozygous loss of function variants in ZNF462 present with a recognizable pattern of phenotype characteristics (Weiss et al., 2017). The first reported case was a reciprocal translocation $\mathrm{t}(2 ; 9)(\mathrm{p} 24 ; \mathrm{q} 32)$ that disrupted both ZNF462 and ASXL2 (Ramocki et al., 2003; Talisetti et al., 2003). This individual presented with ptosis, agenesis of the corpus callosum, ventricular septal defect, periventricular nodular heterotopia, retina and iris colobomas, and a dysplastic left ear and hearing loss. ASXL2 was subsequently associated with Shashi-Pena syndrome which presents as macrocephaly, retrognathia, low set ears, hypertelorism, arched eyebrows, intellectual disability, scoliosis, congenital heart disease, and hypotonia (Shashi et al., 2016). The phenotype of the individual in this case report likely resulted from the loss of function of both ZNF462 and ASXL2. Over a decade later, Weiss et al. described 6 individuals from four families with putative loss of function variants and two unrelated individuals with deletions involving adjacent genes (Weiss et al., 2017). The individuals described by Weiss et al. presented with ptosis (100\%), trigonocephaly or metopic ridging (83\%), and developmental delay or autism spectrum disorder (33\%) (Weiss et al., 2017). Subsequently, Cosemans et al. described an individual with a de novo translocation that disrupted ZNF462 and 
KLF12 who presented with clinical features similar to those described by Weiss et al. (Cosemans et al., 2018; Weiss et al., 2017).

Zinc finger protein 462 (ZNF462) is a $\mathrm{C} 2 \mathrm{H} 2$ type zinc finger transcription factor of unknown function (Nagase, Nakayama, Nakajima, Kikuno, \& Ohara, 2001). Although the specific function of this molecule is unknown, animal studies have shown that it plays a vital role in embryonic development. In Xenopus laevis, knockdown expression of Zfp462 disturbs early embryonic development and results in altered cell division during the cleavage stage; this phenotype is rescued with human ZNF462 mRNA (Laurent et al., 2009). In the mouse model, Zfp462 knockout (KO) mice were prenatal lethal and heterozygous knockout mice $\left(\mathrm{Zfp} 462^{+-}\right)$had developmental delay, low body and brain weights, and anxiety-like behaviors with excessive self-grooming behavior (Wang et al., 2017).

In this report, we describe 14 new individuals in addition to the 10 previously reported cases in the medical literature with truncating variants in ZNF462, collectively review the clinical presentation of this syndrome, and test facial analysis technology's ability to diagnose this syndrome.

\section{METHODS}

This article is protected by copyright. All rights reserved. 


\section{Clinical}

The study was approved by National Human Genome Research Institute Institutional Review Board (IRB). Thirteen new individuals in this report with loss of function variants in ZNF462 were diagnosed using whole exome sequencing (WES) in multiple research and commercial labs including GeneDx and Ambry, and one individual (patient 5) was diagnosed by whole genome sequencing. Nine of the fourteen individuals were ascertained through GeneMatcher (Sobreira, Schiettecatte, Valle, \& Hamosh, 2015).

\section{Facial analysis technology}

We performed two binary classification experiments using the Face2Gene Research application (FDNA Inc., Boston, MA), as previously described (Gurovich et al., 2019). Frontal facial 2D images were collected for three cohorts: individuals with ZNF462 loss of function variants, Noonan syndrome, and healthy controls. Noonan syndrome was used as a second control group due the overlapping facial features of ptosis, downslanting palpebral fissures, hypertelorism, and low set ears in a subset of individuals. All facial images were fully de-identified through the use of the DeepGestalt facial analysis (Gurovich et al., 2019). Controls were matched for age, gender, and ethnicity. 


\section{RESULTS \\ Clinical}

Figure 1 shows the single nucleotide variant and small insertion/deletion (indel) locations on ZNF462 for both the 14 newly reported cases and previously reported cases. All variants are predicted to result in loss of function, including a canonical splice variant in patient 6 that is predicted to result in abnormal splicing (Table 1; Figure 1). Most of these variants are in exon 3, which makes up 54\% of the coding region of $Z N F 42$. .

Table 1 summarizes the clinical features of all 24 affected individuals with 96\% of individuals being Caucasian. Seventeen of 21 families (86\%) have de novo variants, the other four families include unknown, mosaic, and autosomal dominant inheritance (Table 1). The two families with autosomal dominant inheritance demonstrated that the ZNF462 variant segregated with the phenotype characterized in this study: patient 5's father had ptosis surgery and patients 15-17 are from the same family, and previously described (Weiss et al., 2017). The one case of mosaicism was in the mother of patient 1 who had 175 reference reads and 35 alternate reads on WES from a peripheral blood sample [alternate allele frequency $=$ 
$35 /(35+175)=17 \%]$, compared to 120 reference reads and 79 alternate reads in the proband [alternate allele frequency $=79 /(79+120)=40 \%$ ]. The majority of individuals had developmental delay (79\%) and 33\% reported autism spectrum disorders (Table 1). The most common facial features were ptosis (83\%), down slanting palpebral fissures (58\%), exaggerated Cupid's bow/wide philtrum (54\%), arched eyebrows (50\%), and short upturned nose with bulbous tip (46\%). Feeding issues (50\%) and hypotonia (50\%) were common. Less than half of affected individuals reported metopic ridging or craniosynostosis (38\%) or dysgenesis of the corpus callosum (25\%). Less common characteristics included structural heart defects (21\%) and minor limb anomalies (25\%). The clinical analysis of the individuals in this study was heterogenous and not all individuals received brain and heart imaging (Supplementary Clinical Information), thus the above fractions may be an underestimation of brain and heart malformations. Figure 2 shows facial images of individuals with loss of function variants in ZNF462.

\section{Facial analysis technology}

Binary comparison between individuals with loss of function variants in ZNF462 and controls was resulted in two statistics: the mean results involved the computation of the average of the AUC of each of the 10 results, and secondly, the aggregated results consist of a score distribution curve and a receiver-operating-characteristic (ROC) curve for the aggregated results for each photo used in the validation set. 
The binary comparison between ZNF462 $(n=21)$ and healthy controls $(n=21)$ yielded an AUC of 0.96 (STD 0.03), demonstrating good separation between these two cohorts (Supplementary Table 1). Similarly, the comparison between the ZNF462 cohort $(n=21)$ and the Noonan syndrome cohort $(n=16)$ yielded an AUC of 0.97 (STD 0.02) which is also good separation (Supplementary Table 1). The aggregated binary comparison for the ZNF462 group versus health controls yielded an AUC of $0.955(\mathrm{P}=0.006)$ and for the $Z N F 462$ group versus health Noonan syndrome yielded an AUC of $0.972(\mathrm{P}=0.001)$ (Supplementary Figure 1).

Applying DeepGestalt, the confusion matrix/multi-class comparison of the 58 frontal images of the ZNF462 group and both control groups yielded a mean accuracy of 82.88\% (STD 11.79\%) which is significantly better than randomly expected (36.21\%).

\section{DISCUSSION}

We report 24 individuals with loss of function variants in ZNF462 which includes 14 previously unpublished individuals and 10 individuals reported in the medical literature. Based on this larger assembled cohort of individuals, the phenotype of loss of function in ZNF462 is now a distinct multiple congenital anomaly syndrome. We show that ptosis (83\%), developmental delay (79\%), and down slanting 
palpebral fissures (58\%) are three most reported phenotypic features (Table 1). In the previous case series of 6 families and 8 individuals, metopic ridging/craniosynostosis (63\%) was a major phenotypic feature. In this report, we show that metopic ridging/craniosynostosis is still important, but less prevalent (25\%) in this syndrome. Consistent with the previous report by Weiss et al. 2017 (Table 1: patients 15-17), loss of function in ZNF462 appears to have variable expressivity and complete penetrance as demonstrated by patient 5 in the present study with a paternally inherited variant and a father requiring surgery for his ptosis (Table 1; Supplementary Clinical Information). Facial analysis technology was able to accurately differentiate individuals with loss of function in ZNF462 from Noonan syndrome and healthy controls. We predict that the widespread use of facial analysis technology will result in an increase in the number of cases diagnosed this syndrome.

Based on the prevalence of developmental delay, corpus callosum anomalies, congenital heart defects, and hearing loss, we recommend a comprehensive multidisciplinary evaluation of individuals with loss of function variants in ZNF462. This evaluation includes at a minimum: a developmental evaluation, a cardiac exam with echocardiography, brain imaging, hearing evaluation, and consultation with a clinical geneticist and genetic counselor. Other evaluations specific to an 
individual's presentation such as neurosurgery consultation for craniosynostosis may be appropriate. At this time, treatment of complications associated with ZNF462 related syndrome are not different from the general population. As more individuals are studied, future specific management recommendations for ZNF462 related syndrome may be needed.

Pathogenicity of variants in ZNF462 is presumed to be haploinsufficiency based on individuals having loss of function variants only, and this is reinforced by theGenome Aggregation Database (gnomAD) constraint metric of observed/expected loss of function (o/e) value (Karczewski et al., 2019). Values less than 0.35 (o/e) are considered under selection against LOF (https://gnomad.broadinstitute.org) and ZNF462 is well below this threshold with an o/e value of $0.03(90 \% \mathrm{Cl}, 0.01-0.09)$. As noted in the introduction, ZNF462 is important to embryonic development in multiple species. ZNF462 contains $23 \mathrm{C} 2 \mathrm{H} 2$-type zinc finger domains, making DNA binding a likely function (Chang, Stoykova, Chowdhury, \& Gruss, 2007). We now know that ZNF462 is involved in chromatin remodeling. Using histone peptide pull down assays in mouse brain and kidney, Eberl et al. showed that ZNF462 binds H3K9me3, identifying Znf462 as a chromatin reader involved in heterochromatin modification (Eberl, Spruijt, Kelstrup, Vermeulen, \& Mann, 2013). Additionally, Eberl et al. report an interaction with Heterochromatin Protein $1 \alpha(\mathrm{HP} 1 \alpha)$ (Eberl et al., 
2013). As hallmarks of heterochromatin, $\mathrm{HP} 1 \alpha$ and $\mathrm{H} 3 \mathrm{~K} 9 \mathrm{me} 3$ are critical for transcriptional silencing of gene and repetitive DNA and for the maintenance of genome integrity (Almouzni \& Probst, 2011; Beisel \& Paro, 2011; Ren \& Martienssen, 2012), further supporting ZNF462's role in chromatin remodeling. Masse et al. used short hairpin RNA knockdown of pluripotent mouse cells, demonstrating a disruption of pericentromeric domains and redistribution of HP1 $\alpha$ proteins, giving evidence that $Z n f 462$ is instrumental in maintaining heterochromatin in pluripotent cells (Masse et al., 2010).

In summary we present 24 individuals with loss of function variants in ZNF462, and we define a multiple congenital anomaly syndrome that is recognizable from phenotype elements and by using facial analysis technology.

\section{ACKNOWLEDGEMENTS}

We thank the affected individuals and their families for participating in this study. This project was supported by the National Human Genome Research Institute's Intramural Research Program (P.K. and M.M.); NEI grant R01EY027421 and NHLBI grant X01HL132377 (E.C.E), and a CIHR Foundation Grant (FDN-154279) (K.M.B.). The authors have no conflict of interest to declare 


\section{DATA SHARING}

The data that support the findings of this study are available from the corresponding author upon reasonable request.

\section{REFERENCES}

Almouzni, G., \& Probst, A. V. (2011). Heterochromatin maintenance and establishment: lessons from the mouse pericentromere. Nucleus, 2(5), 332338. doi:10.4161/nucl.2.5.17707

Beisel, C., \& Paro, R. (2011). Silencing chromatin: comparing modes and mechanisms. Nat Rev Genet, 12(2), 123-135. doi:10.1038/nrg2932

Chang, Y. S., Stoykova, A., Chowdhury, K., \& Gruss, P. (2007). Graded expression of Zfp462 in the embryonic mouse cerebral cortex. Gene Expr Patterns, 7(4), 405-412. doi:10.1016/j.modgep.2006.11.009

Cosemans, N., Vandenhove, L., Maljaars, J., Van Esch, H., Devriendt, K., Baldwin, A., ... Peeters, H. (2018). ZNF462 and KLF12 are disrupted by a de novo translocation in a patient with syndromic intellectual disability and autism spectrum disorder. Eur J Med Genet. doi:10.1016/j.ejmg.2018.02.002

Eberl, H. C., Spruijt, C. G., Kelstrup, C. D., Vermeulen, M., \& Mann, M. (2013). A map of general and specialized chromatin readers in mouse tissues

This article is protected by copyright. All rights reserved. 
generated by label-free interaction proteomics. Mol Cell, 49(2), 368-378. doi:10.1016/j.molcel.2012.10.026

Gurovich, Y., Hanani, Y., Bar, O., Nadav, G., Fleischer, N., Gelbman, D., . . Gripp, K. W. (2019). Identifying facial phenotypes of genetic disorders using deep learning. Nat Med, 25(1), 60-64. doi:10.1038/s41591-018-0279-0

Karczewski, K. J., Francioli, L. C., Tiao, G., Cummings, B. B., Alföldi, J., Wang, Q., ... \& Gauthier, L. D. (2019). Variation across 141,456 human exomes and genomes reveals the spectrum of loss-of-function intolerance across human protein-coding genes. BioRxiv, 531210.

Laurent, A., Masse, J., Omilli, F., Deschamps, S., Richard-Parpaillon, L., Chartrain, I., \& Pellerin, I. (2009). ZFPIP/Zfp462 is maternally required for proper early Xenopus laevis development. Dev Biol, 327(1), 169-176.

doi:10.1016/j.ydbio.2008.12.005

Lek, M., Karczewski, K. J., Minikel, E. V., Samocha, K. E., Banks, E., Fennell, T., . . . Exome Aggregation, C. (2016). Analysis of protein-coding genetic variation in 60,706 humans. Nature, 536(7616), 285-291. doi:10.1038/nature19057

Masse, J., Laurent, A., Nicol, B., Guerrier, D., Pellerin, I., \& Deschamps, S. (2010). Involvement of ZFPIP/Zfp462 in chromatin integrity and survival of P19 pluripotent cells. Exp Cell Res, 316(7), 1190-1201.

doi:10.1016/j.yexcr.2010.02.024

This article is protected by copyright. All rights reserved. 
Nagase, T., Nakayama, M., Nakajima, D., Kikuno, R., \& Ohara, O. (2001). Prediction of the coding sequences of unidentified human genes. XX. The complete sequences of 100 new cDNA clones from brain which code for large proteins in vitro. DNA Res, 8(2), 85-95. doi:10.1093/dnares/8.2.85

Ramocki, M. B., Dowling, J., Grinberg, I., Kimonis, V. E., Cardoso, C., Gross, A., . . . Millen, K. J. (2003). Reciprocal fusion transcripts of two novel Zn-finger genes in a female with absence of the corpus callosum, ocular colobomas and a balanced translocation between chromosomes 2p24 and 9q32. Eur J Hum Genet, 11(7), 527-534. doi:10.1038/sj.ejhg.5200995

Ren, J., \& Martienssen, R. A. (2012). Silent decision: HP1 protein escorts heterochromatic RNAs to their destiny. EMBO J, 31(15), 3237-3238. doi:10.1038/emboj.2012.172

Shashi, V., Pena, L. D., Kim, K., Burton, B., Hempel, M., Schoch, K., . . Kortum, F. (2016). De Novo Truncating Variants in ASXL2 Are Associated with a Unique and Recognizable Clinical Phenotype. Am J Hum Genet, 99(4), 991-999. doi:10.1016/j.ajhg.2016.08.017

Sobreira, N., Schiettecatte, F., Valle, D., \& Hamosh, A. (2015). GeneMatcher: a matching tool for connecting investigators with an interest in the same gene. Hum Mutat, 36(10), 928-930. doi:10.1002/humu.22844

This article is protected by copyright. All rights reserved. 
Talisetti, A., Forrester, S. R., Gregory, D., Johnson, L., Schneider, M. C., \& Kimonis, V. E. (2003). Temtamy-like syndrome associated with translocation of $2 p 24$ and 9q32. Clin Dysmorphol, 12(3), 175-177. doi:10.1097/01.mcd.0000072161.33788.56

Wang, B., Zheng, Y., Shi, H., Du, X., Zhang, Y., Wei, B., . . Xu, X. (2017). Zfp462 deficiency causes anxiety-like behaviors with excessive self-grooming in mice. Genes Brain Behav, 16(2), 296-307. doi:10.1111/gbb.12339

Weiss, K., Wigby, K., Fannemel, M., Henderson, L. B., Beck, N., Ghali, N., . . . Kruszka, P. (2017). Haploinsufficiency of ZNF462 is associated with craniofacial anomalies, corpus callosum dysgenesis, ptosis, and developmental delay. Eur J Hum Genet, 25(8), 946-951.

doi:10.1038/ejhg.2017.86

This article is protected by copyright. All rights reserved. 
Kruszka Paul (Orcid ID: 0000-0003-4949-0875)

Bedeschi Maria Francesca (Orcid ID: 0000-0003-0174-3562)

Martinez-Agosto Julian (Orcid ID: 0000-0001-6776-6949)

Boycott Kym (Orcid ID: 0000-0003-4186-8052)

Table 1. Phenotype characteristics of individuals with loss of function variants in ZNF462

\begin{tabular}{|c|c|c|c|c|c|c|c|c|c|c|c|c|c|c|c|c|c|c|c|c|}
\hline Patient & Age & Sex & $\begin{array}{l}\text { ZNF462 variant } \\
\text { (NM_021224.5) }\end{array}$ & Inheritance & DD & ASD & Ptosis & $\begin{array}{c}\text { Down } \\
\text { slanting } \\
\text { palpebral } \\
\text { fissures }\end{array}$ & $\begin{array}{l}\text { Arched } \\
\text { eyebrows }\end{array}$ & $\begin{array}{l}\text { Short upturned } \\
\text { nose with } \\
\text { bulbous tip }\end{array}$ & $\begin{array}{l}\text { Exaggerated } \\
\text { cupid } \\
\text { bow/wide } \\
\text { philtrum }\end{array}$ & $\begin{array}{l}\text { Feeding } \\
\text { issues }\end{array}$ & $\begin{array}{l}\text { Epicanthal } \\
\text { folds }\end{array}$ & Ears & $\begin{array}{l}\text { Craniosynostosis } \\
\text { /metopic ridging }\end{array}$ & Hypotonia & Hypertelorism & $\begin{array}{c}\text { Corpus } \\
\text { callosum } \\
\text { dysgenesis }\end{array}$ & $\mathrm{CHD}$ & $\begin{array}{c}\text { Limb } \\
\text { anomalies } \\
\text { (minor) }\end{array}$ \\
\hline 1 & $16 \mathrm{~m}$ & M & $\begin{array}{l}c .2590 \mathrm{C}>\top \\
\text { p. }\left(\mathrm{Arg} g 64^{*}\right)\end{array}$ & $\begin{array}{l}\text { maternal } \\
\text { (mosaic) }\end{array}$ & motor/speech & - & + & - & + & - & + & + & + & low set & - & + & + & normal MRI & $\begin{array}{c}\text { not } \\
\text { reported }\end{array}$ & $\begin{array}{l}\text { fitth finger } \\
\text { clinodactyl } \\
y\end{array}$ \\
\hline 2 & $10 y$ & $\mathrm{M}$ & $\begin{array}{c}\text { c.2542del } \\
\text { p.(Cys848Valfs*66) }\end{array}$ & de novo & motor/speech & + & + & + & + & + & + & + & + & - & - & - & + & normal MRI & $\begin{array}{c}\text { not } \\
\text { reported }\end{array}$ & $\begin{array}{l}\text { not } \\
\text { reported }\end{array}$ \\
\hline 3 & $6 y$ & M & $\begin{array}{c}\text { c.831_834del } \\
\text { p.(Arg277Serfs²6) }\end{array}$ & de novo & motor/speech & - & - & - & - & + & - & + & - & $\begin{array}{c}\text { inner ear } \\
\text { malformation }\end{array}$ & - & + & + & normal MRI & $\begin{array}{l}\text { bicuspidd } \\
\text { aoritc } \\
\text { valve; } \\
\text { VSD } \\
\end{array}$ & $\begin{array}{l}\text { not } \\
\text { reported }\end{array}$ \\
\hline 4 & $\begin{array}{l}2 y \\
7 m\end{array}$ & M & $\begin{array}{c}\text { c. } 6214 \text { _6215del } \\
\text { p.(His2072Tyrfs*8) }\end{array}$ & de novo & speech & - & + & - & - & - & + & + & + & small, lowset & + & - & - & not tested & $\begin{array}{l}\text { not } \\
\text { reported }\end{array}$ & $\begin{array}{c}\text { not } \\
\text { reported }\end{array}$ \\
\hline 5 & $14 y$ & $\mathrm{~F}$ & $\begin{array}{l}\text { c. } 763 \mathrm{C} C \mathrm{~T} \\
\text { p. }\left(\mathrm{Arg} 255^{*}\right)\end{array}$ & paternal & $\begin{array}{l}\text { IEP/special } \\
\text { education }\end{array}$ & - & + & - & + & - & - & - & + & hearing loss & + & - & - & not tested & $\begin{array}{l}\text { not } \\
\text { reported }\end{array}$ & $\begin{array}{l}\text { not } \\
\text { reported }\end{array}$ \\
\hline 6 & $7 \mathrm{~m}$ & $\mathrm{~F}$ & c.7057-2A>G & de novo & $\begin{array}{l}\text { early intervention } \\
\text { for DD }\end{array}$ & - & + & + & + & + & + & + & + & $\begin{array}{l}\text { horizontal crus } \\
\text { helix }\end{array}$ & - & + & + & normal MRI & VSD & $\begin{array}{c}\text { prominent } \\
\text { creases on } \\
\text { hands and } \\
\text { feet } \\
\end{array}$ \\
\hline 7 & $13 y$ & M & $\begin{array}{c}\text { c.6794dup } \\
\text { p.(Tyr2265*) }\end{array}$ & de novo & $\begin{array}{l}\text { cognitive } \\
\text { impairment }\end{array}$ & + & - & - & + & - & + & - & - & $\begin{array}{c}\text { prominent } \\
\text { earslear } \\
\text { pitsthearing } \\
\text { loss } \\
\end{array}$ & + & - & - & not tested & $\begin{array}{l}\text { not } \\
\text { reported }\end{array}$ & $\begin{array}{l}\text { not } \\
\text { reported }\end{array}$ \\
\hline 8 & $2 y$ & $M$ & $\begin{array}{c}\text { c.882dup } \\
\text { p.(Ser295GInfs64) }\end{array}$ & de novo & speech delay & - & + & + & - & - & - & - & - & - & - & - & - & ACC & $\begin{array}{c}\text { not } \\
\text { reported }\end{array}$ & $\begin{array}{l}\text { not } \\
\text { reported }\end{array}$ \\
\hline 9 & $15 y$ & $\mathrm{M}$ & 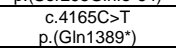 & de novo & global & - & + & + & + & - & + & + & - & lowset & - & + & - & not tested & $\begin{array}{l}\text { not } \\
\text { reported }\end{array}$ & $\begin{array}{l}\text { not } \\
\text { reported }\end{array}$ \\
\hline 10 & $8 y$ & M & $\begin{array}{l}\text { c.1234 } 1235 \text { insAA; } \\
\text { p. }(\text { Ser412*) }\end{array}$ & unknown & $\begin{array}{l}\text { speech delay; } \\
\text { motor apraxia; } \\
\text { IEP }\end{array}$ & - & + & - & - & - & - & + & & $\begin{array}{l}\begin{array}{c}\text { mildly cupped } \\
\text { ears }\end{array} \\
\end{array}$ & - & - & - & normal MRI & $\begin{array}{l}\text { not } \\
\text { reported }\end{array}$ & $\begin{array}{c}\text { not } \\
\text { reported }\end{array}$ \\
\hline 11 & $\begin{array}{c}2 y_{5} \\
m\end{array}$ & $\mathrm{~F}$ & 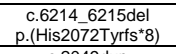 & de novo & - & - & + & - & - & - & - & + & & & - & + & - & not tested & $\begin{array}{l}\text { not } \\
\text { reported }\end{array}$ & $\begin{array}{c}\text { not } \\
\text { reported }\end{array}$ \\
\hline 12 & $9 m$ & $\mathrm{M}$ & $\begin{array}{c}\text { c.2049dup } \\
\text { p.(Pro684Serfs } 14 \text { ) }\end{array}$ & de novo & motor & - & + & + & + & + & + & - & + & - & - & + & + & normal MRI & $\begin{array}{c}\text { not } \\
\text { reported }\end{array}$ & $\begin{array}{c}\text { not } \\
\text { reported } \\
\end{array}$ \\
\hline 13 & \begin{tabular}{|l}
$8 y$ \\
$7 m$
\end{tabular} & M & $\begin{array}{c}c .6631 \text { del } \\
\left.\text { p. Arg2211Glyfs }{ }^{*} 59\right)\end{array}$ & de novo & - & - & + & + & - & + & + & - & - & - & - & - & + & not tested & $\begin{array}{c}\text { not } \\
\text { reported }\end{array}$ & $\begin{array}{l}\text { 5th finger } \\
\text { clinodactyl }\end{array}$ \\
\hline 14 & $8 y$ & $\mathrm{~F}$ & $\begin{array}{l}\text { c.2695G }>\mathrm{T} ; \\
\text { p.(Glu899*) }\end{array}$ & unknown & $\begin{array}{c}\begin{array}{c}\text { cognitive } \\
\text { impairment }\end{array} \\
\text { (n) }\end{array}$ & - & - & - & + & - & - & + & - & - & - & + & - & normal MRI & $\begin{array}{l}\text { not } \\
\text { reported }\end{array}$ & $\begin{array}{c}\text { not } \\
\text { reported }\end{array}$ \\
\hline $15^{a}$ & $2 y$ & $\mathrm{~F}$ & 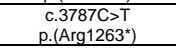 & paternal & - & - & + & + & + & + & + & $\begin{array}{l}\text { not } \\
\text { reported }\end{array}$ & - & - & + & - & + & $\begin{array}{c}\text { ACC; } \\
\text { colpocephaly }\end{array}$ & $\begin{array}{l}\text { not } \\
\text { reported }\end{array}$ & $\begin{array}{l}\text { not } \\
\text { reported }\end{array}$ \\
\hline $16^{a}$ & $4 y$ & $\mathrm{~F}$ & $\begin{array}{l}\text { c. } 3787 \mathrm{C}>\mathrm{T} \\
\text { p. }(\text { Arg1263*) }\end{array}$ & paternal & - & - & + & + & - & + & + & $\begin{array}{c}\text { not } \\
\text { reported }\end{array}$ & + & - & + & - & - & $\begin{array}{c}\text { normal } \\
\text { prenatal } \\
\text { ultrasound }\end{array}$ & $\begin{array}{c}\text { not } \\
\text { reported }\end{array}$ & $\begin{array}{c}\text { not } \\
\text { reported }\end{array}$ \\
\hline $17^{\mathrm{a}}$ & $34 y$ & $\mathrm{M}$ & $\begin{array}{c}\text { c.3787C>T } \\
\text { p.(Arg1263*) }\end{array}$ & maternal & - & - & + & - & - & - & - & $\begin{array}{l}\text { not } \\
\text { reported }\end{array}$ & - & - & + & - & - & not tested & $\begin{array}{c}\text { not } \\
\text { reported } \\
\end{array}$ & $\begin{array}{c}\text { not } \\
\text { reported } \\
\end{array}$ \\
\hline $18^{a}$ & $2 y$ & M & $\begin{array}{l}\text { c.2979_2980delinsA } \\
\text { p.(Val994Trpfs } 1477)\end{array}$ & de novo & speech & + & + & + & + & + & - & + & + & $\begin{array}{l}\text { left overfolded } \\
\text { ear }\end{array}$ & + & - & - & - normal MRI & $\begin{array}{l}\text { not } \\
\text { reported }\end{array}$ & $\begin{array}{c}\text { single } \\
\text { palmar } \\
\text { crease; } ; 5^{\text {th }} \\
\text { finger } \\
\text { clinodactyl } \\
y \\
y\end{array}$ \\
\hline $19^{\mathrm{a}}$ & $32 \mathrm{~m}$ & $M$ & $\begin{array}{c}\text { c. } 4263 \mathrm{del} \\
\text { p.(Glu1422Serfs }{ }^{*} 6 \text { ) }\end{array}$ & de novo & motor/speech & + & + & + & - & + & 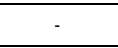 & - & + & lowset & + & + & + & $\begin{array}{c}\text { hypoplastic } \\
\text { corpus }\end{array}$ & D-TGA & $\begin{array}{c}\text { not } \\
\text { reported }\end{array}$ \\
\hline
\end{tabular}

This article is protected by copyright. All rights reserved. 


\begin{tabular}{|c|c|c|c|c|c|c|c|c|c|c|c|c|c|c|c|c|c|c|c|c|}
\hline & & & & & & & & & & & & & & & & & & $\begin{array}{l}\begin{array}{c}\text { callosum and } \\
\text { venticuloume } \\
\text { galy }\end{array} \\
\end{array}$ & & \\
\hline $20^{\mathrm{a}}$ & $5 y$ & $\mathrm{~F}$ & 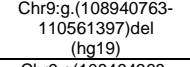 & de novo & - & . & + & + & + & + & + & $\begin{array}{c}\text { not } \\
\text { reported }\end{array}$ & + & & - & + & - & $\begin{array}{c}\text { hypoolasasic } \\
\text { corlus } \\
\text { calosum }\end{array}$ & $\begin{array}{l}\text { not } \\
\text { tested }\end{array}$ & $\begin{array}{l}\text { not } \\
\text { reported }\end{array}$ \\
\hline $21^{a}$ & ${ }_{15 y}$ & $F$ & 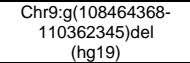 & de novo & motorfintellectual & + & . & . & 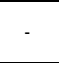 & . & + & $\begin{array}{c}\text { not } \\
\text { reported }\end{array}$ & - & & - & - & + & not tested & vSD & $\begin{array}{c}\text { not } \\
\text { reported }\end{array}$ \\
\hline $22^{2}$ & 9y & M & 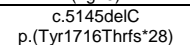 & de novo & $\begin{array}{c}\text { motorlspeech } \\
\text { delay }\end{array}$ & + & + & - & - & - & - & $\begin{array}{l}\text { not } \\
\text { reported }\end{array}$ & - & - & - & + & . & normal MRI & $\begin{array}{c}\text { not } \\
\text { reported }\end{array}$ & $\begin{array}{c}\text { not } \\
\text { reported }\end{array}$ \\
\hline $23^{b}$ & $5 y$ & $\mathrm{~F}$ & 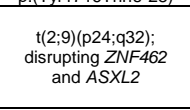 & de novo & $\begin{array}{l}\text { intellectual } \\
\text { disability }\end{array}$ & + & + & + & + & + & + & + & - & $\begin{array}{l}\text { lowset; hearing } \\
\text { loss }\end{array}$ & - & + & - & $\begin{array}{l}\text { ACC; dilated } \\
\text { venricles }\end{array}$ & 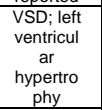 & $\begin{array}{c}\text { single } \\
\text { palmar } \\
\text { crease; } \\
\text { hypoplastic } \\
\text { finger nails }\end{array}$ \\
\hline $24^{\circ}$ & $24 y$ & м & 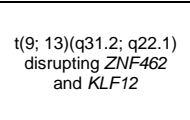 & de novo & $\begin{array}{l}\text { intellectual } \\
\text { disability }\end{array}$ & + & + & + & - & - & . & + & + & lowset & + & + & - & $\begin{array}{l}\text { hypoplastic } \\
\text { corpus } \\
\text { callosum }\end{array}$ & $\begin{array}{l}\text { none } \\
\text { reported }\end{array}$ & $\begin{array}{c}\text { Small } \\
\text { hands and } \\
\text { feet; } \\
\text { proximally } \\
\text { praced } \\
\text { phums }\end{array}$ \\
\hline & & & & & $79 \%$ & $33 \%$ & $83 \%$ & $58 \%$ & $50 \%$ & $46 \%$ & $54 \%$ & $50 \%$ & $46 \%$ & $50 \%$ & $38 \%$ & $50 \%$ & $25 \%$ & $25 \%$ & $21 \%$ & $\begin{array}{l}\text { thumbs } \\
25 \% \\
\end{array}$ \\
\hline
\end{tabular}

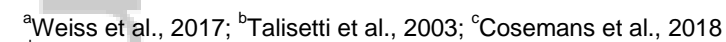

In order to avoid overestimating phenotype prevalence, we divided each positive phenotype report by the entire cohort $(\mathrm{n}=24)$.

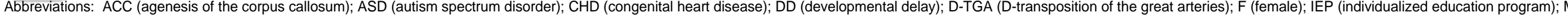
(male); MRI (magnetic resonance imaging); ventricular septal defect (VSD);

This article is protected by copyright. All rights reserved. 
Kruszka Paul (Orcid ID: 0000-0003-4949-0875)

Bedeschi Maria Francesca (Orcid ID: 0000-0003-0174-3562)

Martinez-Agosto Julian (Orcid ID: 0000-0001-6776-6949)

Boycott Kym (Orcid ID: 0000-0003-4186-8052)

\section{FIGURE LEGENDS}

Figure 1. ZNF462 variant locations. Variants from the present study are in blue and variants from previous publications are shown in black. Thirteen of the seventeen variants are on exon 3 which makes up 54\% of ZNF462. Note that there are two unrelated individuals with the p.(His2072Tyrfs*8) variant (patients 4 and 11 in Table 1).

Figure 2. A. Patient 1; B. Patient 2; C. Patient 3; D. Patient 4; E. Patient 5 at 8 years; F. Patient 6 at two and 7 months; G. Patient 7; H. Patient 8 at 3 months and 2.5 years; I. Patient 9 at ages 8 and 15 years; J. Patient 12; K. Patient 13; L. Patient 14; M. Patient 15; N. Patient 16; O. Patient 17; P. Patient 18; Q. Patient 19; R. Patient 20; S. Patient 21; T. Patient 22; U. Patient 24; (Figures M-T are from Weiss et al., 2017 and Figure $U$ is from Cosemans et al., 2018) 


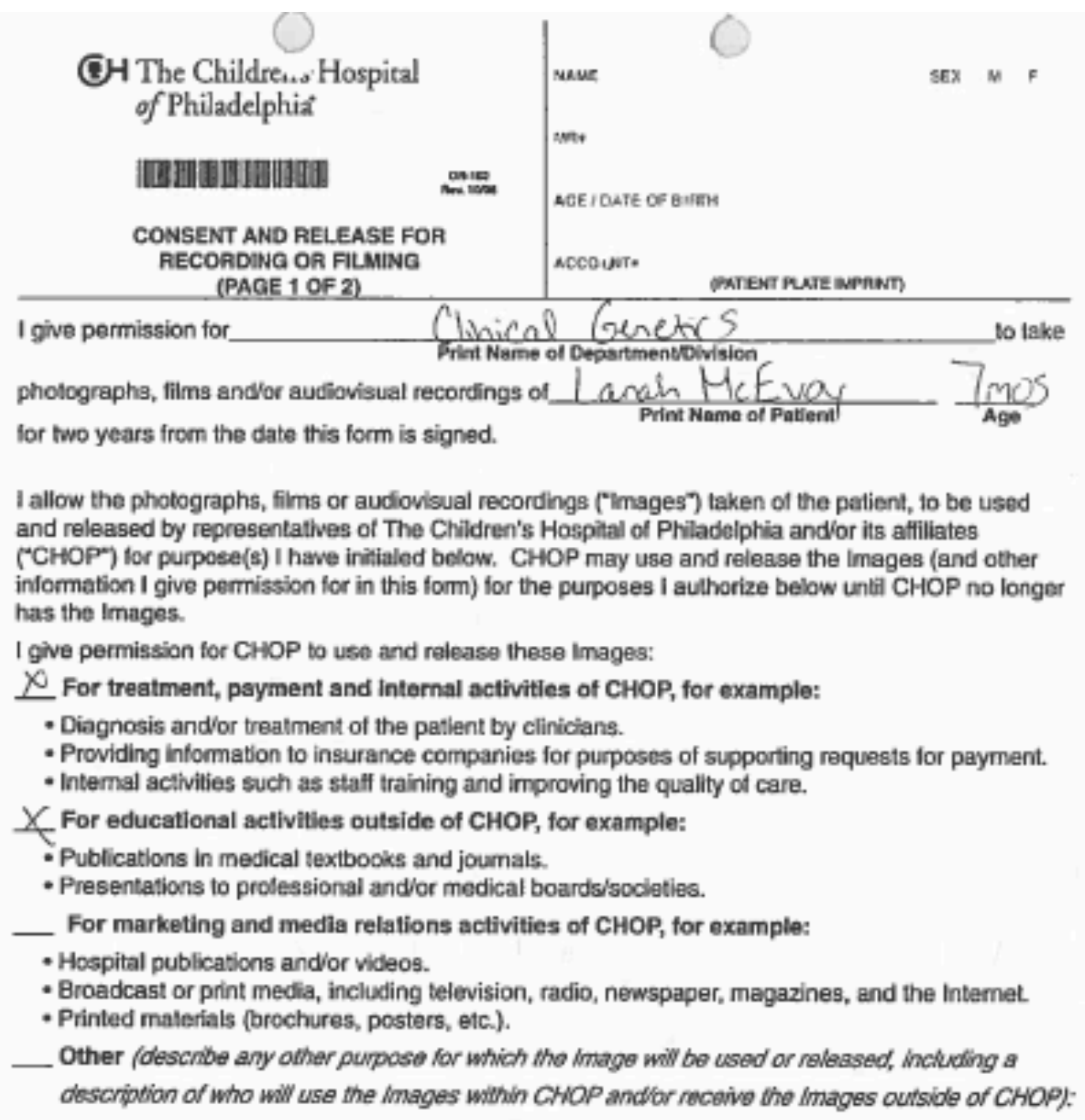

Permission to Use and Release Patient Name and Other Information with the Images: Some Images themselves may identify the patient (for example, pictures that show a patient's face). In some cases, CHOP may wish to use or release the patient's name along with the Images. Please initial one of the following for use of the patient's name with the Image:

I agree to the use and/or release of the patient's name with the Images.

X I do not agree to the use andior release of the patient's name with the Imagas.

I also allow the following patient information to be used andior released along with the images: 


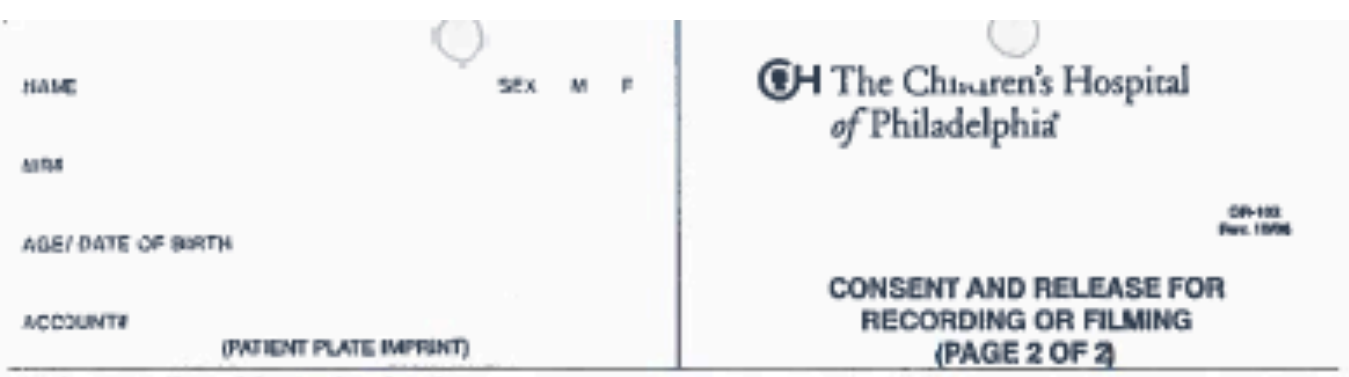

Understanding this Authorization:

- Wy decision to sign this authorization will not usualy affect the patient's ablity to got care at CHOP. A times, CHOP may be required to record or film the petient to provide modical services. If I do not agree to the recording or filming in these situations, services may not be provided.

- I understand that CHOP may use and release Images as the law requires or allows without further permission from the patient, even it I do not agree. See the CHOP Notice of Privacy Practices for datails about how medical information, including Images, may be used and shared without further permission from the patient -- http:/Ww/w.chop.edu/about_chop/hipaa/npp.shtml

- Aler signing this form, I can change my mind and ask that recording or filming stop. If I change my mind after recoeding or filming is done, I can notily the CHOP Department / Division indicated above in writing to ask that the Images not be used or released. This will not change any use or release of the Images by CHOP before receiving my notice. If the Images were released cutside of CHOP, I understand the Images may continue to be used even after I withdraw my permission lor CHOP to use or share them.

- IfI allow CHOP to release Images to other individuals or organizations, I understand that the recipients could use, distribute, broadeast and/or publish them in ways that do not protect the patient's privacy and that CHOP cannot control.

- The Images belong to CHOP. I will not be paid for the use or release of the Images.

By sgning. I understand that I am authorizing CHOP to take, use and release Imagas of the palient as dencribed above.

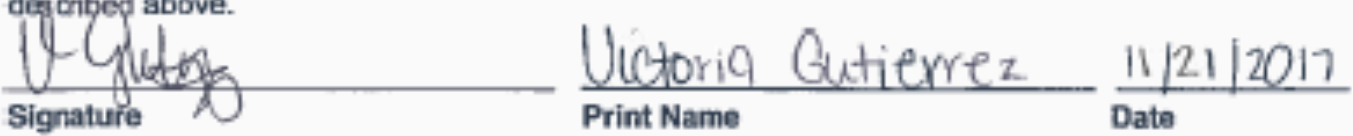

Relationship to Patlent: ЈPatlent $\not 3$,Parent $\square$ Legal Guardian $\sqsupset$ Other:

\section{TO BE COMPLETED bY CHOP STAFF}

Brief description of Image: Face

Indicate type of Images taken: BWPhotograph $\square$ Film $\square$ Audio Fecording $\square$ Other:

Print name of ipdividual taking the Images and indicate affiliation with CHOP by checking below: Carey McDaraci

\$CCHOP Employee $\square$ Contractor $\sqsupset$ News Media $\square$ Other

Print name of Hospital representative superylsing the recording:
Cares.

Locetion where Images will be stored, if other than the patient's medical record: Jines m Cho secured rive 

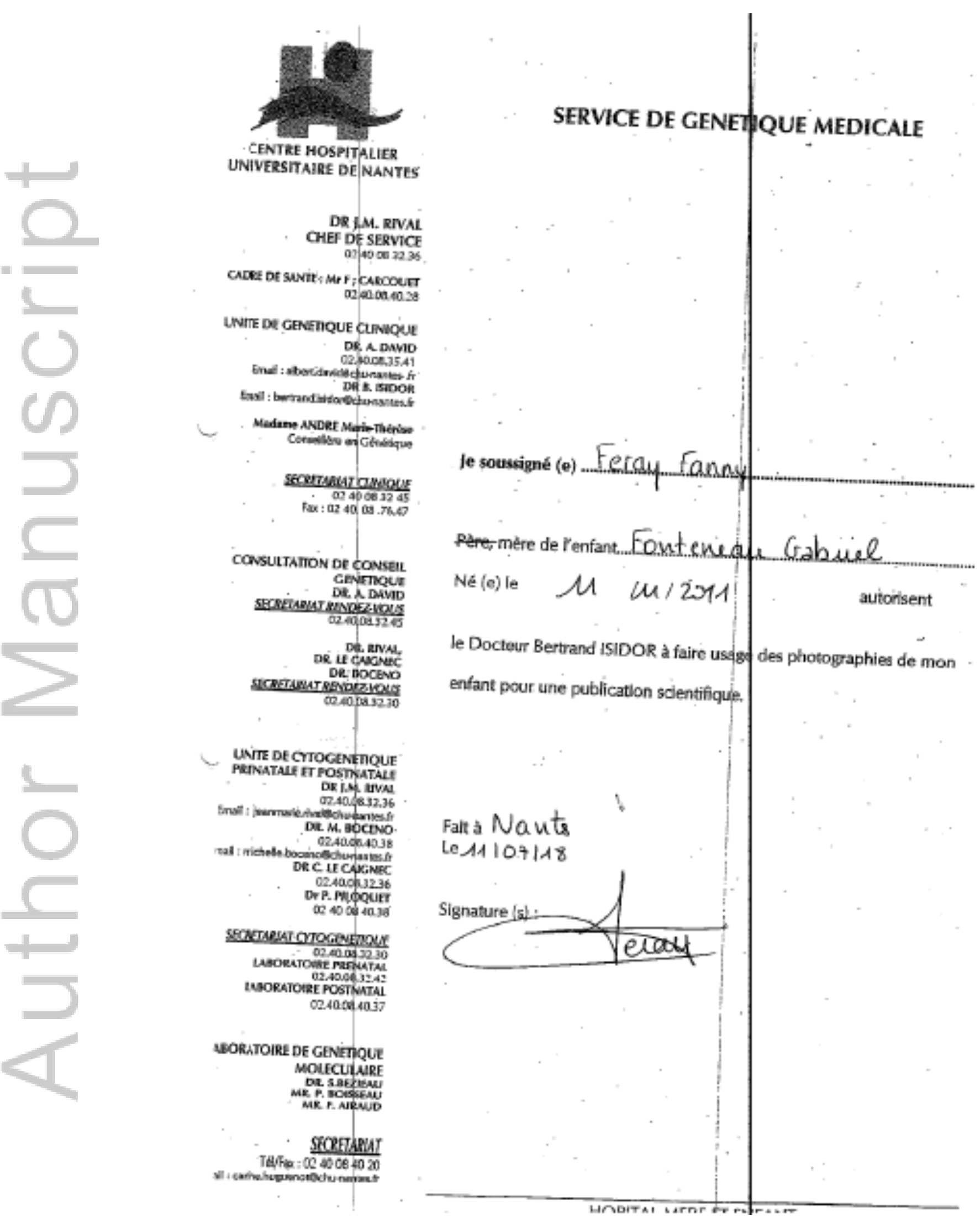

This article is protected by copyright. All rights reserved. 


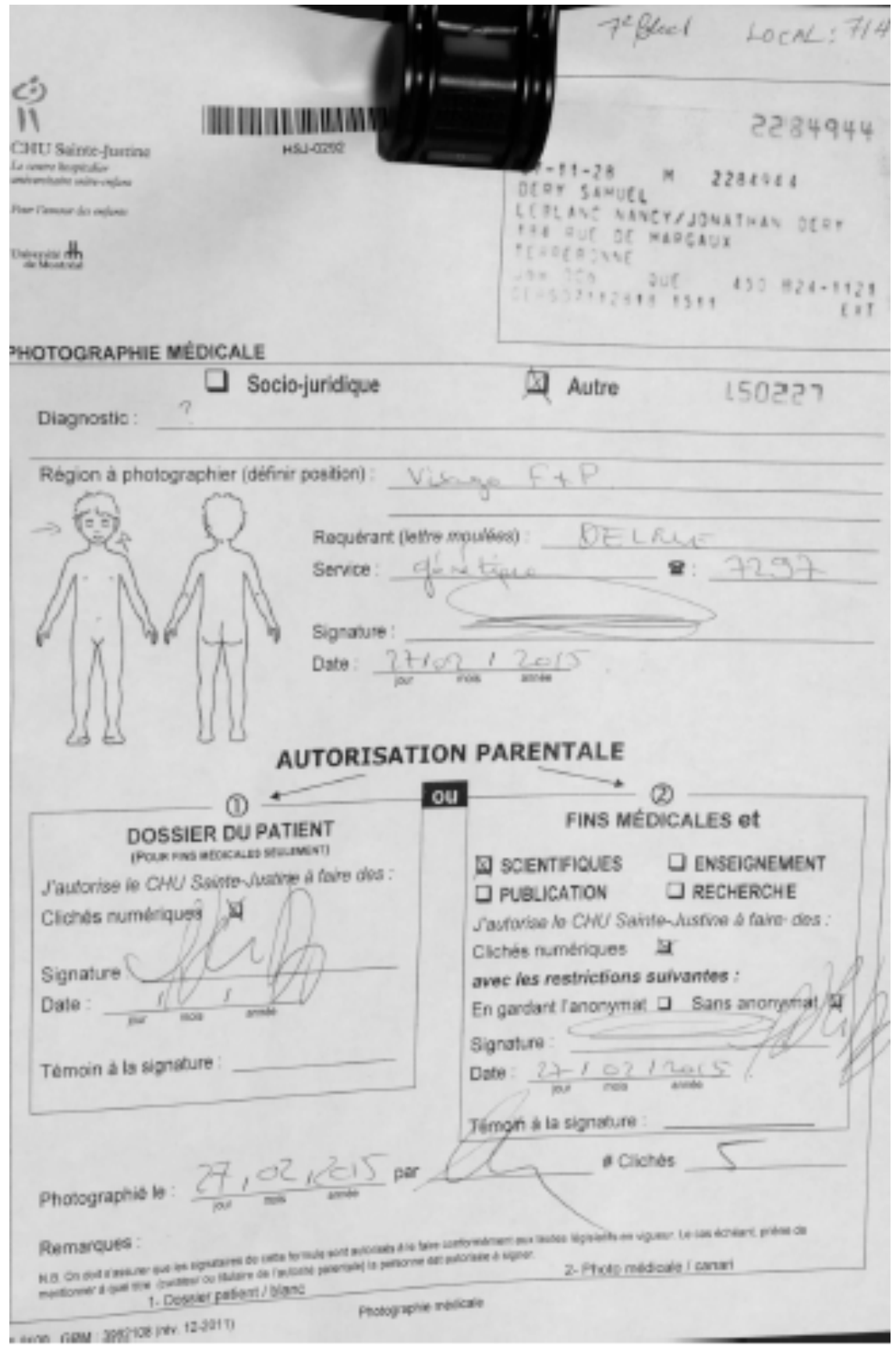

This article is protected by copyright. All rights reserved. 
CHU Saine-Jestise HSد-0202

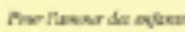

vonganth

PHOTOGRAPHIE MÉDICALE

\section{Diagnostic : \\ Socio-juridique \\ Autre \\ 150227}

$07-11-28 \quad M \quad 2284044$

DEQY SAMUEL

LEGLANC SANCY/JONATHAN OERY

17A QUE OE MAPGAUX

teset a svive

$n=2 \times+$

Due

$430824-1121$

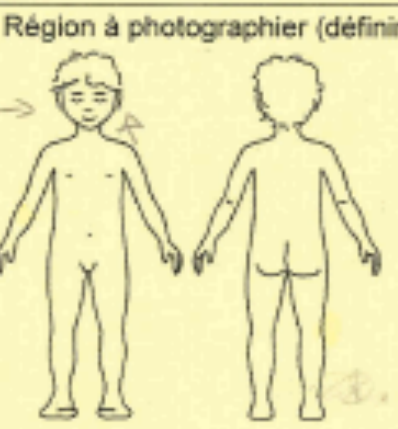

Requérant (lettre moulées)

Service:

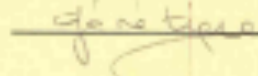

Signature :

IIn

Date :

$$
\frac{23102 / 2015}{\text { bur mos }}
$$

\section{AUTORISATION PARENTALE}

\section{(1)}

\section{DOSSIER DU PATIENT}

(POUn nus ntoncNes seuernenT)

J'autorise le CHU Sainte-Justine a faire des:

Clichés numériques

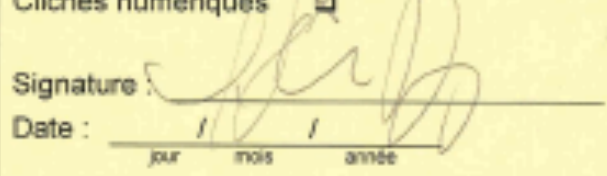

Témoin à la signature :

Tëmoin à la signature:

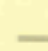

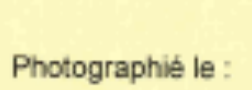

$$
\text { : }
$$

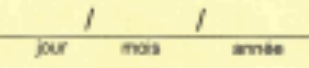
par

ou

-

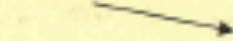

FINS MÉDICALES et

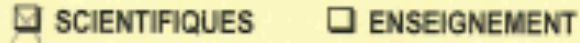

$\triangle$ PUBLICATION $\square$ RECHERCHE

Sautarise le CHU Sainte-Justine a faive des:

Clichés numériques Q

avec les restrictions suivantes:

En gardant ranonymat $\square$ Sans anonymat $\square$

Signature :

Date :

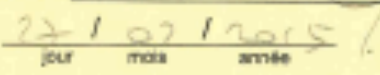

Témoin à la signature : \# Clichés

Remarques :

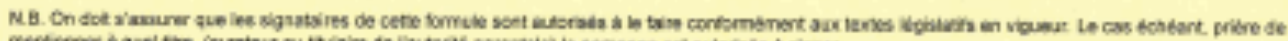

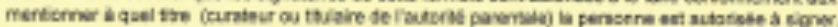
1- Dossier patient / blanc 2. Phate medicale f canari 
Acute Senvices Dlvision

Diagnostics Directorate

West of Scoulasid Regienal Genetic Servioe

Leved $2 \mathrm{~A}$ Laboratory Medicine

Qupen Elazbeth Liverstly Hospital

1345 Gomen Rast

3116

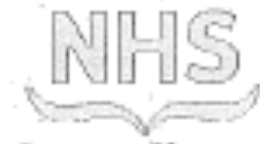

Greater Glasgony

and Clyde

Glaspiom

G51 4TF

\section{CONSENT FORM FOR PHOTOGRAPHY/AUDIO/MDEO RECORDING OF PATIENTS}

I consent to photograph/si/fudioffuidev recording/s) being taken for aw/himy child's persenal medical record for diagnostic purposes anly

I consent to photograph(s)/atadioffvideo-recording(s) being taken formminmy child's personal medical record for diagnostic purposes, and to shoan to appropsiate health protesslonals and students, in the UX

ict and abroad, to aid medical teaching and mosearch

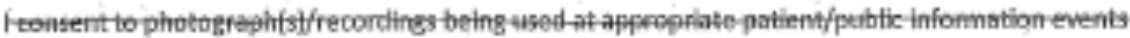

I understand that I have the fieht to withdraw consent ary time by contacting the West of Scotiand Genetic Services.

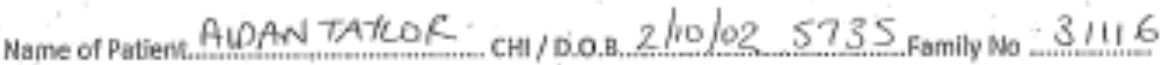

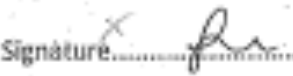

Relation to patient (patient/parent/guardian)

Date 24.108118

I consent to my/my child's photograph[s] being published in an open access joumal, textbook or other form of medical publication (which mas include the internet), and therefore may be seen tw the general public. as wail 75 medical professionals.

I understand that once published, it will not be possible to completely withdraw this consent.

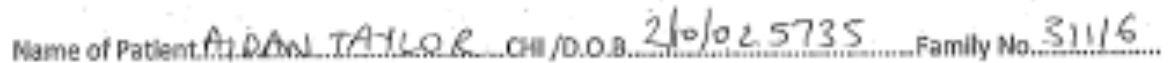

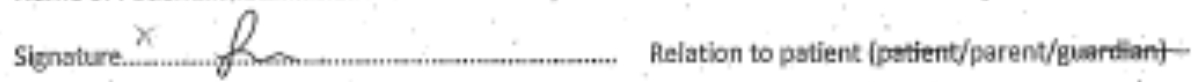

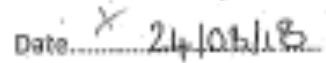

Name and signature of medical professlonal requesting illustratiuns/recording and obtainire consent

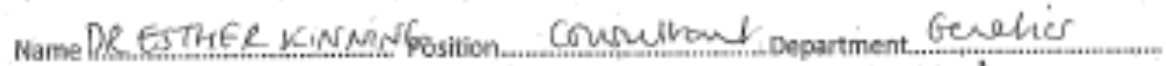

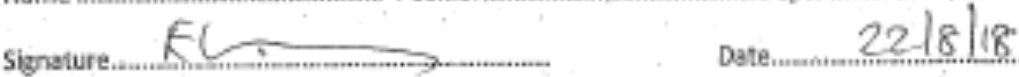

Versian 1 Saptember 2016

For review March 2017 
Patient aame: BRI-ANNA THOMPSON Date: $\operatorname{Mar} 13 / 19$ check here if minor or unable to provide consent

I consent for medieal photographs to be made of me or my child (or person for whom I am legal guardian). I understand that the information may be used in my medical record, for parposes of medical teaching, or for publication in medical textbooks or joumals as I have designated below. By consenting to these medical photographs I understand that I will not receive payment from any party. Refusal to consent to photographs will in no way affect the medical care I will receive. If I have any questions or wish to withdraw my consent in the future I may contact:

Dr. Paul Kruszka, NIH, 301-402-9654, paul.kruszkagnih.gov

By signing this form below I confirm that this consent form has been explained to me in terms which I understand.

1) I consent for these photographs to be used in medicat pablications, including medical joumals, textbooks, and electronic publications. I understand that the image may be seen by members of the general public, in addition to seientists and medical researchers that regularly use these publications in their professional education. Although these photographs will be used without identifying information such as my name, I understand that it is possible that someone may recognize me. I also agree for my image to be shown for teaching parposes and to be used for my medieal record.

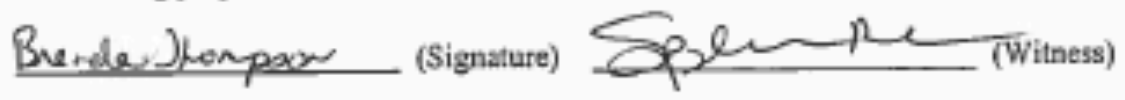

2) I agree for my image to be shown for tenching purposes AND to be used for my medical record but NOT FOR medical publication:

(Signature) (Witness)

3) I agree to use of my image for medical reconds ONLY:

(Signature) (Witness)

For patients between ages 7 and 18 years, a signature below indicates that the information in this consent form has been explained to me, and I assent to use of my images as outlined above:

(Signature of patient)

(Witness)

This article is protected by copyright. All rights reserved. 
Department of Genetics

Lundiaan 6

WKZ - KCO4 0842

PO Box 85090, 3508 AB Utrecht

The Netherlands

Tel. +31897553800

Fax. +318875 5380

7107691

26-01-2015

M

The undersigned, parent(s) of:

name:

date of birth:

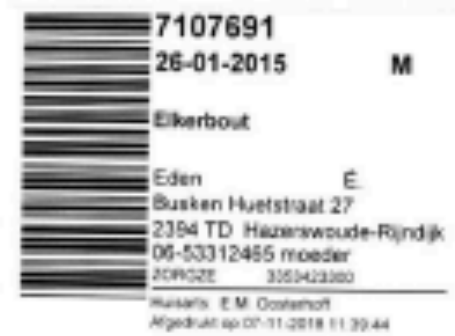

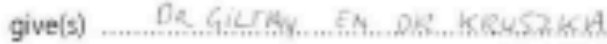

permission to publsh clinical photographs of their son / daughter* in a medical journal.

Place Hazerswoude Rindyin

Date: $18-11.2018$

Signature (fathert

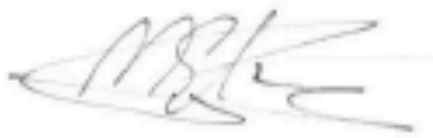

"Please delete as appropriate.
Signature imatherk:

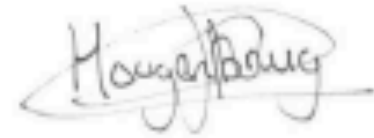

This article is protected by copyright. All rights reserved. 


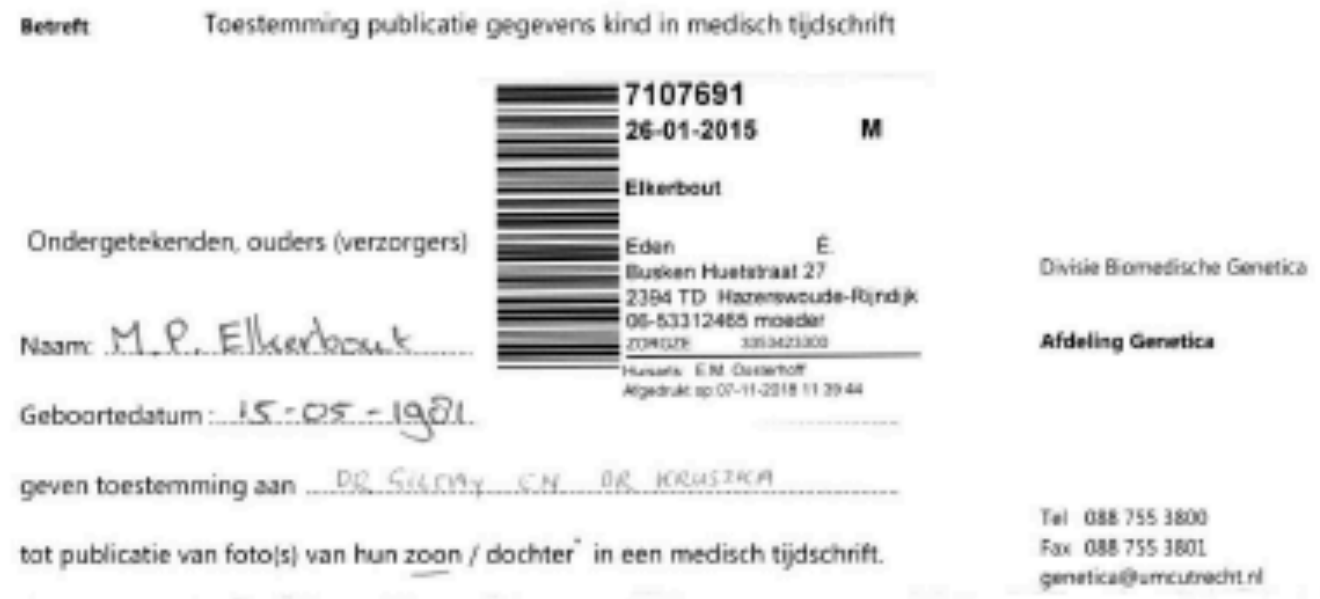

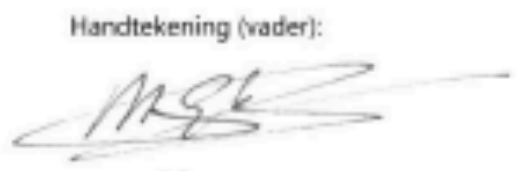

Plaats: Havarswoudc

Datum: $18-11-i 8$

s.v.p. doorhalen wat niet van toepassing is

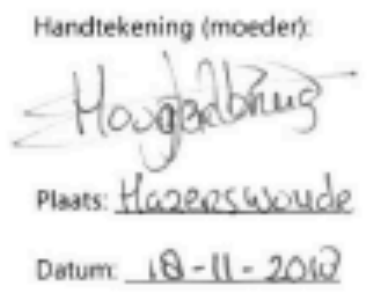

Beuroekabres:

Landian 6

3584 EA Unecht

Pavadres

Huscostnumer $\mathrm{sC4} 0942$

Postous asoco

3509 2日 Ltrecte

wwow umcutrecht in 
Putient name: Tota Riccarid Date: jel 021 zojis

check here if minor or unable to provide consent

I consent for medical photographs to be made of me or my child (or person for whom I am legal guardian). I understand that the information may be used in my medical resord, for purposes of medical teaching, or for publication in medical textbooks or jaumals as I have designated below. By consenting to these medical pbotographs I understand that I will not receive payment from any party. Refusal to consent to phowographs will in no way affect the medical care I will receive. If I have any questions or wish to withdraw my consent in the future I may contact:

Dr. Paul Kruszka, NIH, 301-402-9654, paul.kruszkaønih.gov

By signing this form below I confirm that this consent form has been explained to me in terms which $I$ understand.

1) I consent for these photographs to be used in medical publications, including modical journals, textbooks, and electronic publications. I understand that the image may be seen by mermbers of the general public, in addition to scietsists and medical researchers that regularly use these publications in their professional education. Although these photographs will be used without identifying information such as my name, I understand that it is possible that someone may recognize me. I also agree for my image to be showa for teaching purposes and to be used for my medical record.

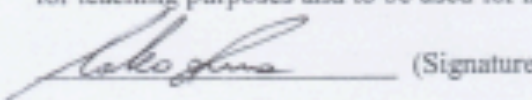

(Witness)

2) I agree for my image to be shown for lesehing purposes AND to be used for my medical record but NOT FOR medical publication:

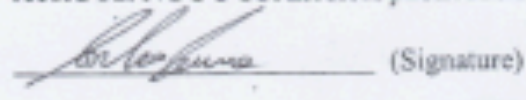

(Witness)

3) I agree to use of my image for medical reconds ONLY:

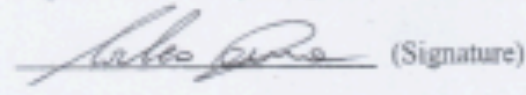

(Witness)

For patients between ages 7 and 18 years, a signature below indicates that the information in this consent form has been explained to me, and 1 assent to use of my images as outlined above

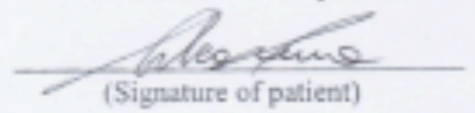

(Witness) 
Patient name: UIADIMYK MAKSMOV Date: $05 / 28 / 2018$

$\square$ chock here if minor or unable tc provide consent

I consent for medical photographs to be made of me or my child (or person for whom 1 ama legal guandian). I understand that the information may be used in my medical record, for parposes of medical teaching, or for publication in medical textbooks or journals as I have desigamed below. By consenting to these modical phetographs I understand that I will not receive payment from any party. Reflusal to consent to photographs will in no way affeet the medical care I will receive. If I have any questions or wish to withdraw my consent in the future I may contact:

Dr. Paul Kruszka, NIH, 301-402-9554, paul.krusaka@nih.gov

By signing this form below I coafim that this consent form has been explained to me in terms which I understand.

1) I consent for these photographs to be used in medical publications, iacluding medical joumals, textbooks, and elestronic publications. I understand that the image may be steen by members of the general public, in addition to seientists and modical researchers that reguiarly use these publicatons in their professional education. Although these photographs will be used without identifying information such as my name, 1 understand that it is possible that someone may recognize me. I also agree for my image to be shown for teaching purposes and to be used for my medical rocord.
impervelve
(Signature)
MHEstow U.
(Witness)

2) I agree for my image to be shown for teaching purposes AND to be used for my metical record but NOT FOR medieal publication:

(Signature)

(Witness)

3) I agree to use of my image for medical records ONLY:

(Signature)

(Witness)

For patients between ages 7 and 18 years, a signature below indicates that the information in this consent form has been explained to me, and I assent to use of my images as outlined above:
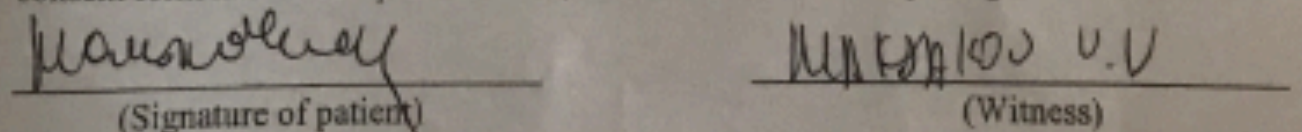

This article is protected by copyright. All rights reserved. 


\section{AUTHORIZATION FOR RELEASE OF PROTECTED HEALTH INFORMATION (PHI): COMMUNICATIONS | MARKETING}

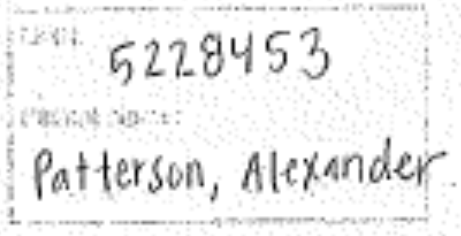

UCLA Health is committed to protecting the privacy of our patients, That is why we must obtain your written consant before we may reveal detalis about you, or your ward's, care.

Participant: Alexander Pattersion Date of Birth:

130,2017

Addreso: 1714 stoner Are Apt 3 los Angelef CA 90025

Phone Number: $(310,993-782.1$ Email (optiona):

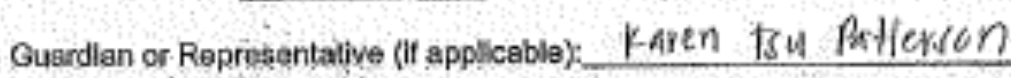

BRIEF DESCRIPTION OF PROJECT:

Nitl vescarchers are publushing a paper documenting the clinical prefentainon and range of pinenctuper in indiaduds with zNF4ez mulations. The autwer wauld uye the dinion information and if posible, phorigraphs, for publication.

HEALTH INFORMATION DISCLOSED MAY INCLUDE (check all that apply):

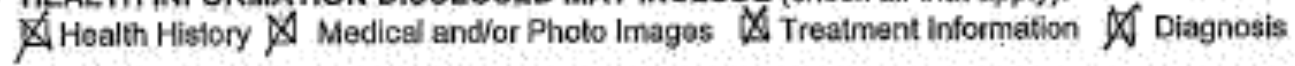

$\square$ Other:

PURPOSE OF PROJECT (check all that apply):

$\square$ Educational Material X Publication $\square$ Media Outreach $\square$ Promotional Material

$\square$ Other:

INFORMATION REGARDING CARE WILL BE GATHERED FROM:

'X. UCLA Health employees involved in the patient's care.

$\square$ Other:

INFORMATION REGARDING CARE WILL BE DISCLOSED TO:

$\square$ UCLA Health $\square$ Outside Media KOther: Natun nal institule of nealth, Dr. PAul

I SPECIFICALLY AUTHORIZE RELEASE OF THE FOLLOWING (if applicable):

Not Applicable

Mental health treatment information NOT includlng psychotherapy notes.

Q HIV test results and/or HIV treatment information

$\square$ Alcohol and/or substance abuse treatment information. 


\section{AUTHORIZATION FOR RELEASE OF PROTECTED HEALTH INFORMATION (PHI): COMMUNICATIONS | MARKETING}

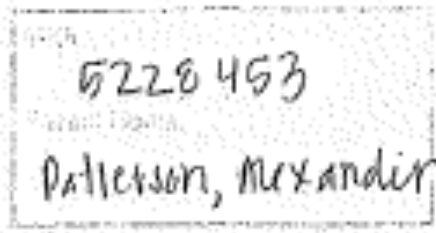

LIMITATIONS ON THE USE OF PARTICIPANT'S HEALTH INFORMATION (please be speciflc):

\section{DURATION OF AUTHORIZATION}

This authorization will remain in effect unta:

If no date is Isted, this authorization will remain in effect for a period of five (5) years from the date of signature attached.

UCLA is required by lew to keep health information confidential. If you have authorized the disclosure of health information to scmeone who is not logally required to keep it confidential, it may be subject to re-disclosure.

\section{DO I HAVE TO SIGN THIS FORM?}

Absolutely notl This authorization to release health information is voluntary. Declining to sign this authorization will not affect you, or your ward's, treatment, payment enrollment, or eilgibility for benefits.

\section{CAN I RECEIVE A COPY OF THIS AUTHORIZATION?}

Yosl You have the right to request and recelve a signed copy of thls authorization.

WHAT IF I CHANGE MY MIND? .

You may revoke this authoization at any time by wriling to:

UCLAHealthNewsịmednet.ucla,edu

OR

UCLA Health Sciences Media Relations

924 Westwood Boulevard, Sulte 350

Los Angeles, CA 90024

Because UCLA Health puts a great deal of time and care into conceiving and developing communications and publications, we ask that you write to the address above as soon as possible after deciding to revoke your authorization.

Revocation will be effective upon receipt, except to the extent that UCLA or others have already relied on it. If the multimedia items have already been shared, it may not be possibla to recall them. 


\section{UGLA Health}

\section{AUTHORIZATION FOR RELEASE OF PROTECTED HEALTH INFORMATION (PHI): COMMUNICATIONS | MARKETING}

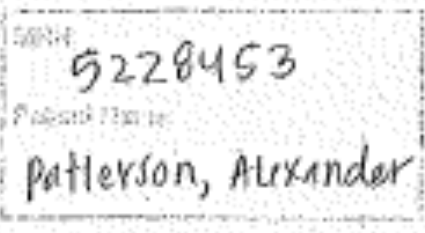

I have read this form and all of my questions have been answered. My signature confirms that 1 understand and accopt all of the above conditions, and approve the disclosure and use of health information by UCL.A Health.

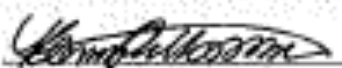

Signature of petlent, parent or conservator

\section{$2 / 7 / 2018$}

Date

12:37 PM

If not signed by patient, indicate relationship or guardlan: Mother

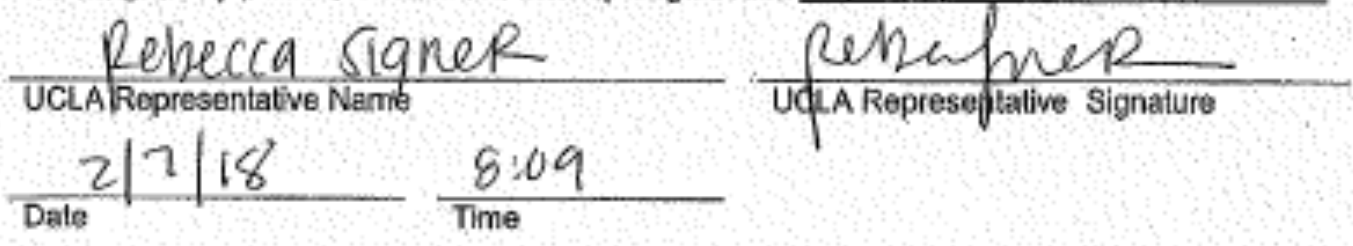

I have securately and completely read this consent to (patlent or patient's legal representative) in the pationt's or logal ropresentative's primary language (Identify language). Helshe understood all of the terms and condifions and acknowledged his/her agreement by signing the document in my presence.

Signature of Translator

Date

Translator ID \# 

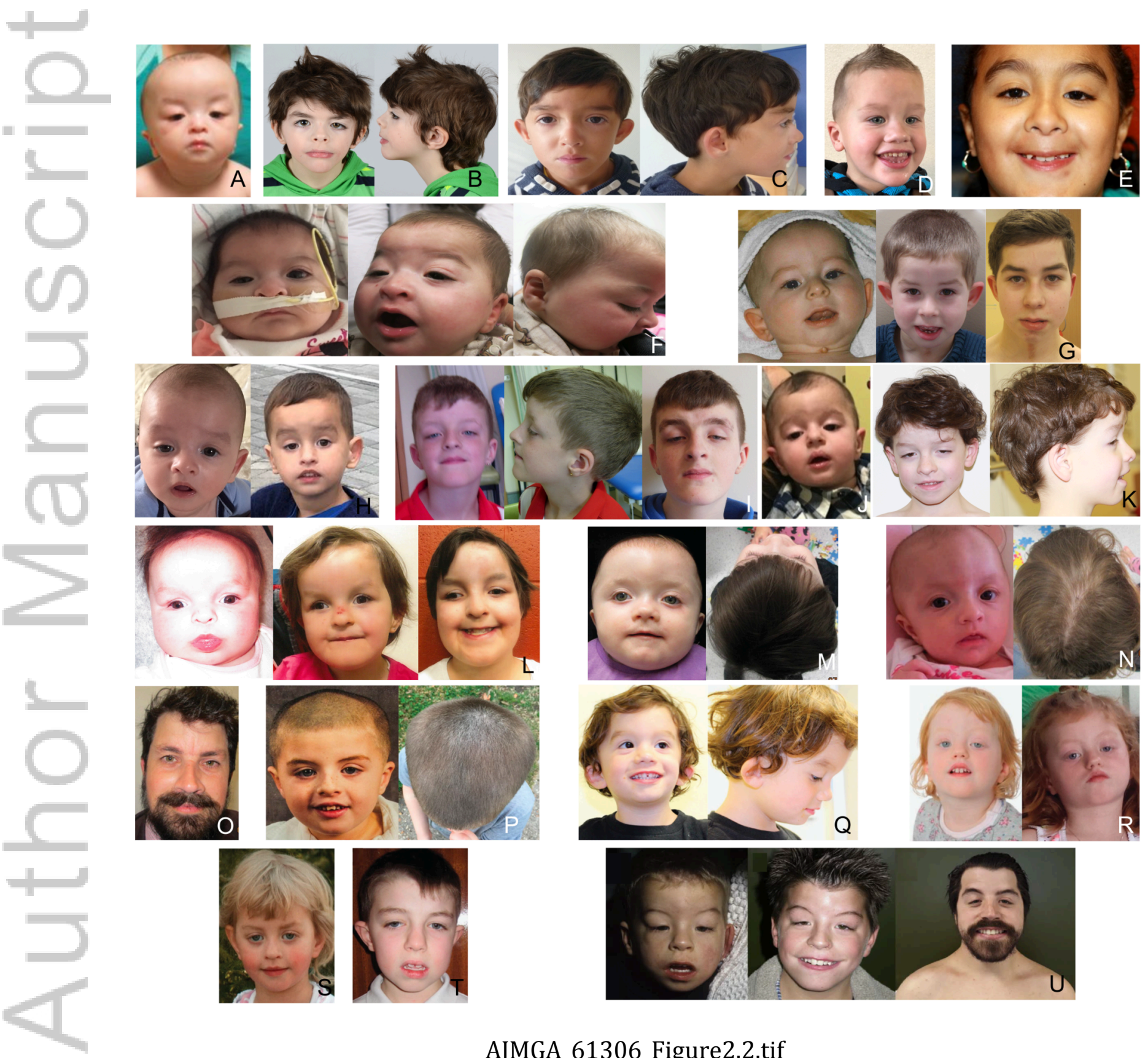

AJMGA_61306_Figure2.2.tif 
Zinc Finger Protein 462 (ZNF462)

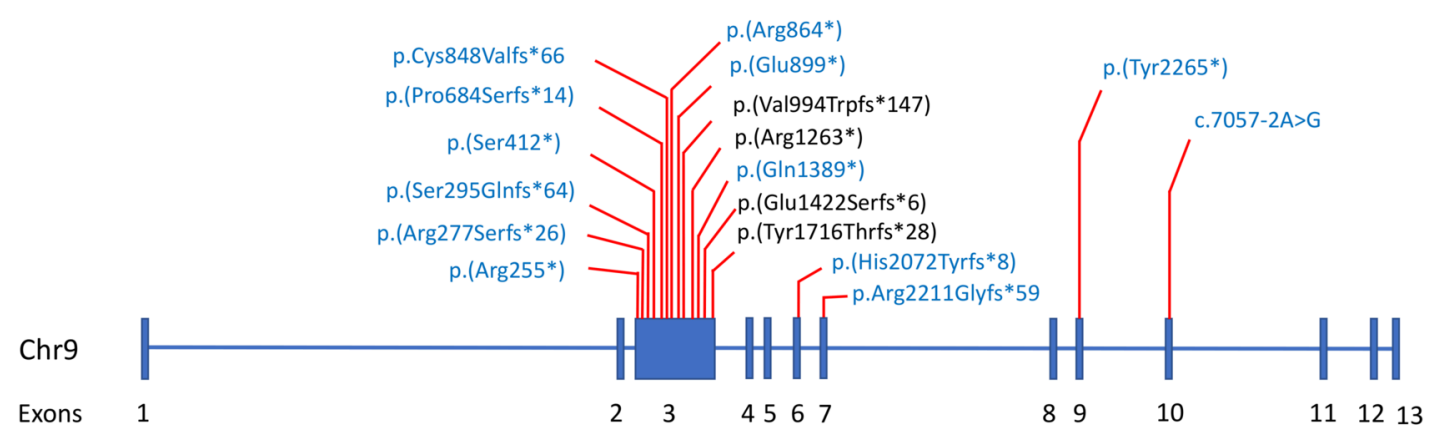

AJMGA_61306_Revision_Figure_1.3.tif

This article is protected by copyright. All rights reserved. 


\section{University Library}

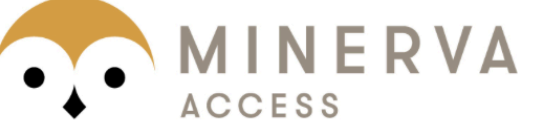

A gateway to Melbourne's research publications

Minerva Access is the Institutional Repository of The University of Melbourne

\section{Author/s:}

Kruszka, P;Hu, T;Hong, S;Signer, R;Cogne, B;Isidor, B;Mazzola, SE;Giltay, JC;van Gassen, KLI;England, EM;Pais, L;Ockeloen, CW;Sanchez-Lara, PA;Kinning, E;Adams, DJ;Treat, K;Torres-Martinez, W;Bedeschi, MF;lascone, M;Blaney, S;Bell, O;Tan, TY;Delrue, MA;Jurgens, J;Barry, BJ;Engle, EC;Savage, SK;Fleischer, N;Martinez-Agosto, JA;Boycott, K;Zackai, EH;Muenke, M

Title:

Phenotype delineation of ZNF462 related syndrome

Date:

2019-07-30

\section{Citation:}

Kruszka, P., Hu, T., Hong, S., Signer, R., Cogne, B., Isidor, B., Mazzola, S. E., Giltay, J. C., van Gassen, K. L. I., England, E. M., Pais, L., Ockeloen, C. W., Sanchez-Lara, P. A., Kinning, E., Adams, D. J., Treat, K., Torres-Martinez, W., Bedeschi, M. F., lascone, M. ,... Muenke, M. (2019). Phenotype delineation of ZNF462 related syndrome. AMERICAN JOURNAL OF MEDICAL GENETICS PART A, 179 (10), pp.2075-2082. https://doi.org/10.1002/ ajmg.a.61306.

Persistent Link:

http://hdl.handle.net/11343/286216 